\title{
The effect of Zhangfei/CREBZF on cell growth, differentiation, apoptosis, migration, and the unfolded protein response in several canine osteosarcoma cell lines
}

\author{
Rui Zhang ${ }^{1,3}$, Douglas H Thamm² and Vikram Misra ${ }^{1 *}$
}

\begin{abstract}
Background: We had previously shown that the bLZip domain-containing transcription factor, Zhangfei/CREBZF inhibits the growth and the unfolded protein response (UPR) in cells of the D-17 canine osteosarcoma (OS) line and that the effects of Zhangfei are mediated by it stabilizing the tumour suppressor protein p53. To determine if our observations with D-17 cells applied more universally to canine OS, we examined three other independently isolated canine OS cell lines_-Abrams, McKinley and Gracie.

Results: Like D-17, the three cell lines expressed p53 proteins that were capable of activating promoters with p53 response elements on their own, and synergistically with Zhangfei. Furthermore, as with D-17 cells, Zhangfei suppressed the growth and UPR-related transcripts in the OS cell lines. Zhangfei also induced the activation of osteocalcin expression, a marker of osteoblast differentiation and triggered programmed cell death.

Conclusions: Osteosarcomas are common malignancies in large breeds of dogs. Although there has been dramatic progress in their treatment, these therapies often fail, leading to recurrence of the tumour and metastatic spread. Our results indicate that induction of the expression of Zhangfei in OS, where p53 is functional, may be an effective modality for the treatment of OS.
\end{abstract}

Keywords: Canine osteosarcoma, Zhangfei/CREBZF, p53, Apoptosis, Osteocalcin

\section{Background}

Osteosarcoma (OS) is the most common primary malignant bone tumour in children and adolescents, although its incidence in dogs is ten times greater than in humans [1]. Spontaneously occurring osteosarcomas in dogs are an ideal model for cancer research due to their anatomical and physiological similarities with human counterparts (reviewed by [2-4]).

We had previously shown that the basic leucine zipper (bLZip) domain-containing transcription factor, Zhangfei/ CREBZF/SMILE inhibits the growth and the unfolded protein response (UPR) in the D-17 canine osteosarcoma (OS) cell line [5] and that the effects of Zhangfei are

\footnotetext{
* Correspondence: vikram.misra@usask.ca

'Department of Microbiology, Western College of Veterinary Medicine,

University of Saskatchewan, Saskatoon, SK, Canada

Full list of author information is available at the end of the article
}

mediated by stabilizing the tumour suppressor protein p53 [6]. To determine if our observations with D-17 cells applied more universally to canine OS, we examined three other independently isolated canine OS cell lines-Abrams, McKinley [7-10] and Gracie [11]. The purpose of this study was to determine the inhibitory role of Zhangfei in these OS cell lines by exploring its potential involvement in growth, differentiation, apoptosis, and metastasis.

Zhangfei was initially identified through its interaction with the host cell factor (HCF1) a protein required for the initiation of herpes simplex virus gene expression [12]. Unlike other bLZip transcription factors, Zhangfei appears to be incapable of binding to consensus bLZip response elements as a homodimer [13]. Instead, it fulfills its role in transcriptional regulation by hetero-dimerizing with and modulating other transcription factors or signaling molecules, such as 
Luman/CREB3 [14], Xbp1 [15], ATF4 [16], SMAD 1,5,8 [17], herpes simplex virus VP16 [18], and p53 [19].

\section{Results and discussion}

All four canine OS cells lines express functional p53

To confirm the effects of Zhangfei we had observed in D-17 OS cells we examined three other canine OS cell lines. We have shown that Zhangfei exerts its effect on cell growth and the UPR by stabilizing p53 [6] and it therefore has no effect on cancer cells that do not possess functional p53. To assess the status of p53 in the canine cell lines we amplified p53 transcripts from the cells using PCR and determined the nucleotide sequences of the products. Figure $1 \mathrm{~B}$ shows the derived amino acid sequences of p53 from the cell lines and the reference sequence from the canine genome database. All four cell lines contained transcripts for p53 that, with the exception of a few amino acid variations, were identical to the reference sequence. None of the amino acid polymorphisms in the sequences were at positions identified as important for p53 function [20] (Figure 1A,B).

To determine if the p53 proteins in the cell lines were functionally active, we transfected the cells with a plasmid that expressed the reporter protein chloramphenicol acetyl transferase (CAT) regulated by a promoter with two copies of a p53 response element (pCATp53RE). As a negative control, cells were transfected with a plasmid (pCAT3B) without the response elements. Parallel cultures were transfected with a plasmid expressing Zhangfei. Figure $1 \mathrm{C}$ shows that expression of CAT was activated in all four cell lines in a p53 response element-dependent manner and that the presence of Zhangfei enhanced expression.

\section{Cellular outcome following ectopic expression of Zhangfei: growth arrest, apoptosis and differentiation} We next compared the effect of Zhangfei on the growth characteristics of Abrams, McKinley, and Gracie cells with its effect on D-17 cells. The cells were infected with adenovirus expressing either Zhangfei (Adeno-ZF) or the control protein $\beta$-galactosidase (Adeno-LacZ). Cell growth was monitored by the WST-1 Cell Proliferation Assay. In agreement with previous results, all four Adeno-ZF-infected cells failed to divide as early as day 1 after infection as determined by their ability to convert WST-1 Cell Proliferation reagent and absorb light at $405 \mathrm{~nm}$. Mock infected cells continued to grow for three days and the growth of Adeno-LacZ-infected cells was indistinguishable from mock-infected cells (Figure 2). Apoptosis was induced in all four cell lines as a result of Zhangfei expression (Figure 3). D-17 and Abrams appeared to be more sensitive as cultures had substantial numbers of apoptotic cells at $24 \mathrm{hr}$. The response in McKinley and Gracie, was slower with cultures showing
20 to $34 \%$ apoptotic cells by $48 \mathrm{hr}$. Since the level of p53 in response to Zhangfei was similar in all the cell lines the quantitative differences in the induction of apoptosis suggests that Zhangfei may have effects that are, at least partially, independent of p53.

Since the effect of Zhangfei was most dramatic on D-17 and Abrams cells we selected them for further analysis. Zhangfei may stop cell growth by inducing differentiation and/or causing apoptosis, we therefore performed a transcript level analysis of the OS differentiation markerosteocalcin [21] in D-17 and Abrams cells infected with either Adeno-ZF or Adeno-LacZ. Compared with LacZ-expressing and even vitamin D3-treated cells (negative and positive controls, respectively), Zhangfei significantly increased the expression of osteocalcin transcripts in a time-dependent manner (Figure 4).

\section{Expression of Zhangfei inhibits the ability of canine osteosarcoma cells to close a scratch wound}

Migratory behaviour in cancer cells is a typical hallmark of malignancy. To investigate whether ectopic expression of Zhangfei correlated with altered migratory behaviour, we performed cell motility assays on the canine OS cultures. Following scratch wounding, wound closure was significantly slower in cultures (D-17 and Abrams canine cells) infected with Adeno-ZF compared to cultures infected with Adeno-LacZ or mock-infected cells (Figure 5), showing that the ectopic expression of Zhangfei indeed causes decreased cell motility in canine osteosarcoma cells. However, it is also possible that the decrease in ability of Zhangfei-expressing cells to grow heal the scratch wound may be because of a decrease in growth rates rather than a decrease in the ability to migrate.

\section{Zhangfei negatively regulates the UPR in canine osteosarcomas}

The unfolded protein response (UPR) is an adaptive cellular stress response that alleviates ER stress or, failing, induces apoptosis. In previous studies, we found Zhangfei was a negative regulator of the UPR in D-17 canine OS cells [5]. To investigate if Zhangfei could consistently suppress the UPR in other canine OS cells, the four canine OS cell lines infected with either Adeno-ZF or Adeno-LacZ were treated with the UPR pharmacological inducer thapsigargin, or were deprived of glucose. The latter treatment is a known physiological inducer of the UPR. Both treatments increased the level of transcripts for UPR transcripts Xbp1s, HERP, CHOP and GRP78 (Figure 6A) and Zhangfei suppressed the transcripts in thapsigargin treated (Figure 6B) and glucose deprived (Figure 6C) cells. In contrast, LacZ had no obvious effect. In addition, this decrease in mRNA was reflected in a decrease in UPR proteins (Xbp1s, HERP, and GRP78) in thapsigargin-treated D-17 


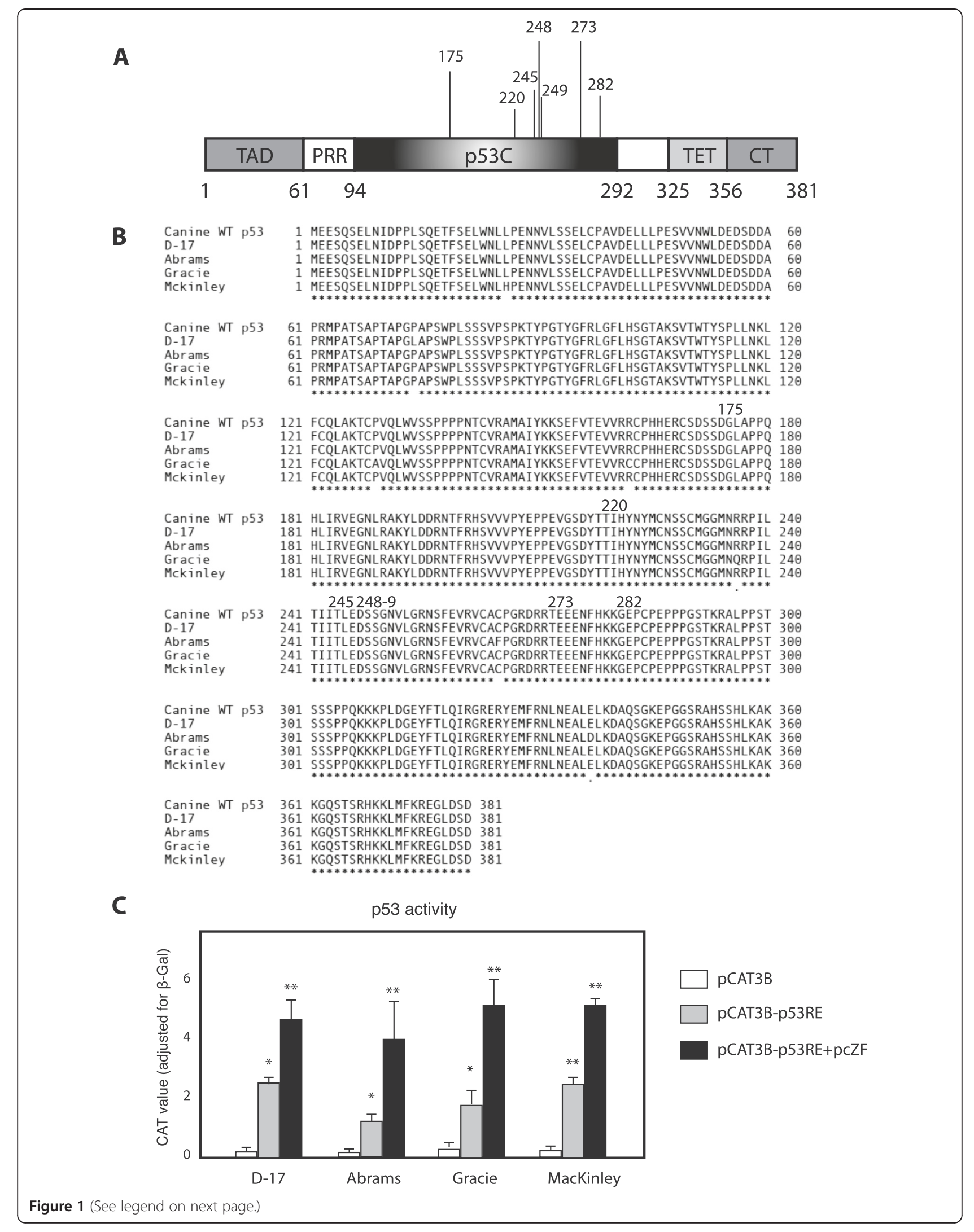


(See figure on previous page.)

Figure 1 p53 in dog osteosarcoma cell lines. (A) Schematic structure of full-length p53. TAD: N-terminal transactivation domain; PRR: proline-rich region; p53C: central DNA-binding domain; TET: tetramerization domain; $C$ : extreme carboxyl terminus. p53C is the domain where most cancerassociated p53 mutations are located. The numbers below the diagram indicate amino acid residues delineating the domains and numbers above the diagram represent the residues with highest frequency of oncogenic missense mutations [20]. (B) Derived amino acid sequence alignment of p53s from 4 dog OS cell lines and dog wild-type p53. The residues that have high mutant frequency were marked above the diagram. Accession numbers: KP279761, KP279762, KP279763, KP279764 (C) p53 proteins of dog OS cell lines have transcriptional activity, and Zhangfei enhances p53-dependent transactivation. D-17, Abrams, McKinley, and Gracie cells were transfected with $0.5 \mu \mathrm{g}$ of pCAT3B or pCAT3B-p53RE, in the presence or absence of $1 \mu \mathrm{g}$ of pcZF. $24 \mathrm{~h}$ after transfection, the CAT activity was determined. Values represented the relative CAT activity (adjusted by $\beta$-galactosidase) of different treatments. Standard deviations from means of three individual experiments are shown. Significance of differences of the means $\left({ }^{*} P<0.05,{ }^{* *} P<0.01\right)$ were determined using ANOVA.

[5] and Abrams (Figure 6D) cells. Figure 6E, which showed intracellular proteins detected by immunofluorescence, also supported these data-the Xbp1s protein was undetectable in D-17 and Abrams cells expressing Zhangfei.

\section{Conclusions}

Canine OS is an aggressive tumour that accounts for approximately $85 \%$ of primary bone tumours in the dog [22]. OS causes local skeletal destruction resulting in osteoproductive and osteolytic lesions, and it is highly metastatic to the lungs. Although there has been dramatic progress in the standard treatments of OS, including amputation, chemotherapy, and palliative radiation therapy, these therapies often fail, leading to recurrence of the tumour and metastatic spread [23-26]. Over the years, combined therapies, such as chemotherapy combined with immune modulators, have been practiced on dog OS $[27,28]$, although with poor overall survival times.

The dog is a well-established model for spontaneous OS in humans, owing to striking similarity in biology and gene expression. The large size of dogs, relative outbreeding, and immunocompetence increase their model potential. Furthermore, dogs with spontaneous tumors
A
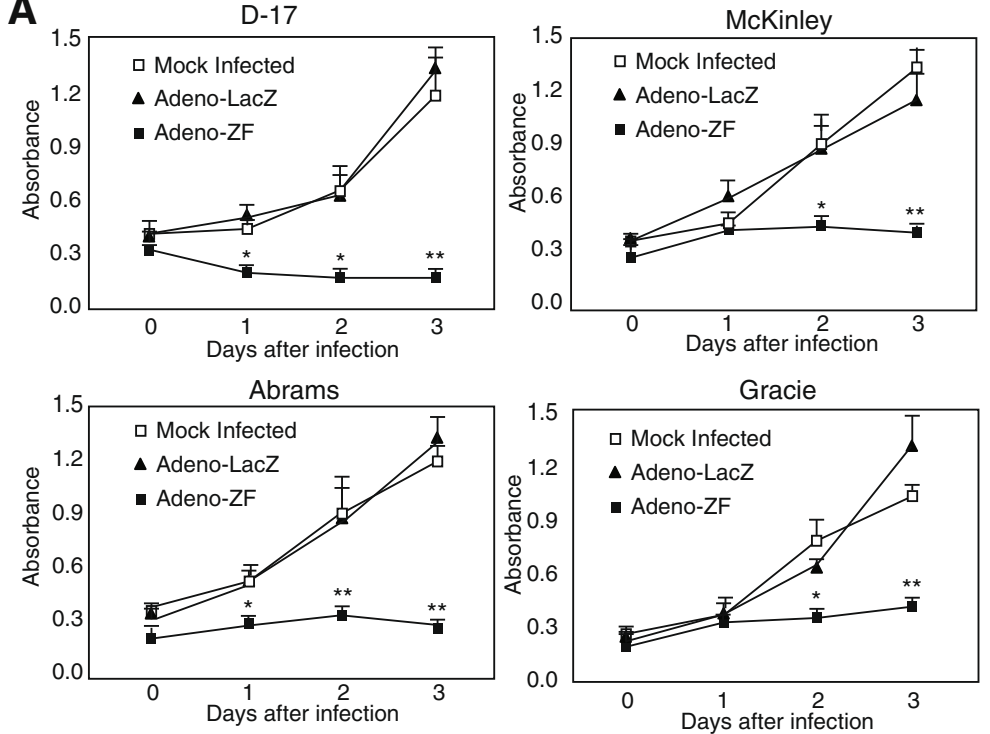

B

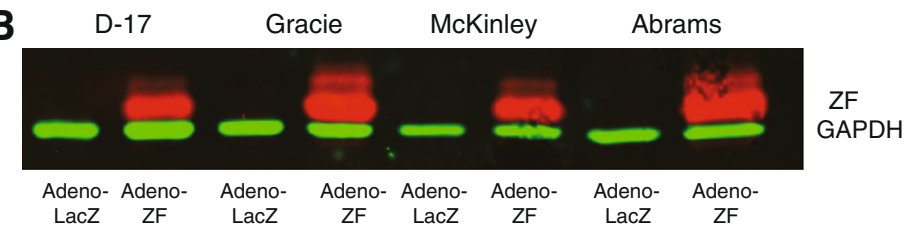

Figure 2 Ectopic expression of Zhangfei suppresses cell growth in canine osteosarcomas. D-17, Abrams, McKinley, and Gracie canine OS cells were mock-infected or infected with adenovirus vectors expressing either Zhangfei (Adeno-ZF) or $\beta$-galactosidase (Adeno-LacZ) and growth rates were measured $(\mathbf{A})$ by absorbance at $405 \mathrm{~nm}$ with WST-1 at different time points after infection. Error bars indicate standard deviations from means of three individual experiments. Standard deviations from means of three individual experiments are shown and significance $\left({ }^{*} P<0.05\right.$, $\left.{ }^{* *} P<0.01\right)$ was determined using ANOVA. (B). Zhangfei was detected by immunoblotting using antiserum against Zhangfei. 


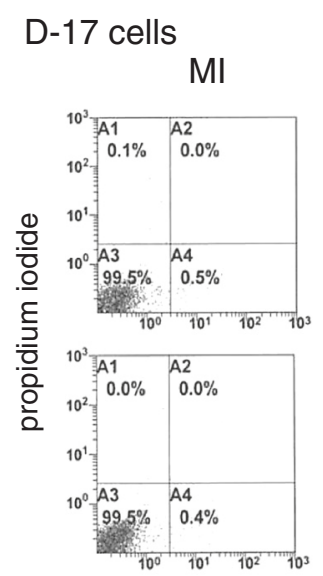

\section{Adeno-LacZ}
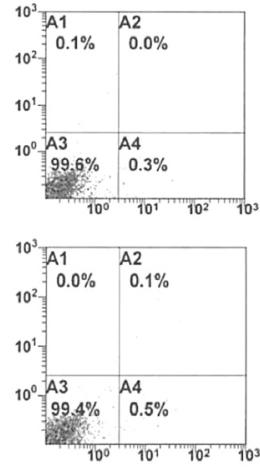

Abrams cells
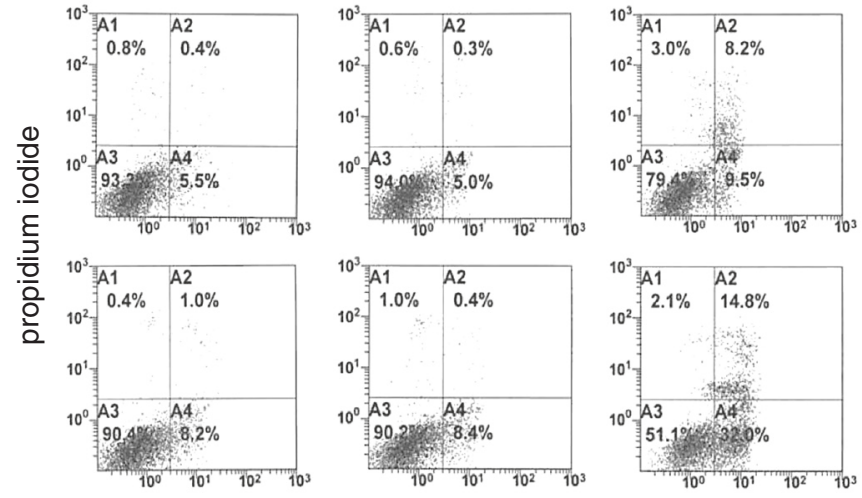

Annexin V

\section{McKinley cells}
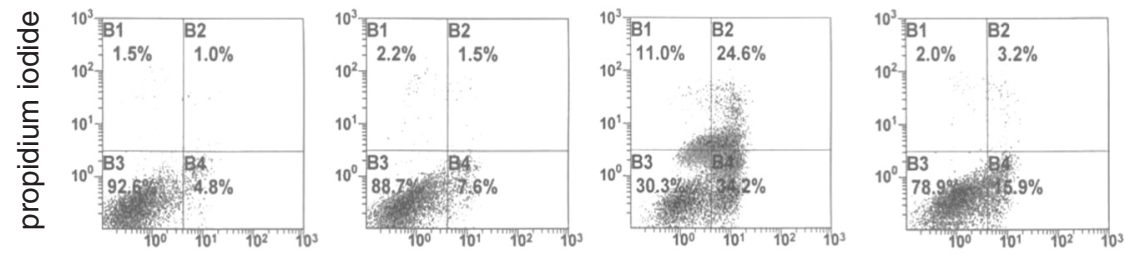

$48 h$

\section{Gracie cells}
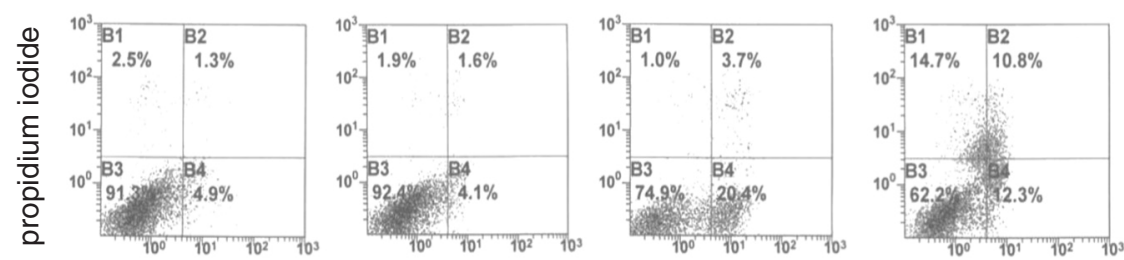

$48 h$

\section{Annexin V}

Figure 3 Zhangfei causes canine osteosarcoma cells to commit apoptosis. D-17, Abrams, McKinley and Gracie cells, mock-infected or infected with Adeno-ZF or Adeno-LacZ or treated with $50 \mu \mathrm{M}$ etoposide (positive control) were stained with fluorescent Annexin V and propidium iodide. Unstained cells or cells staining with either or both dyes were enumerated by FACS. A4 represents the percentage of total cells undergoing apoptosis. For McKinley and Gracie only reading for $48 \mathrm{hr}$ are shown. Differences between LacZ and ZF-expressing cells were more pronounced at that time-point. 

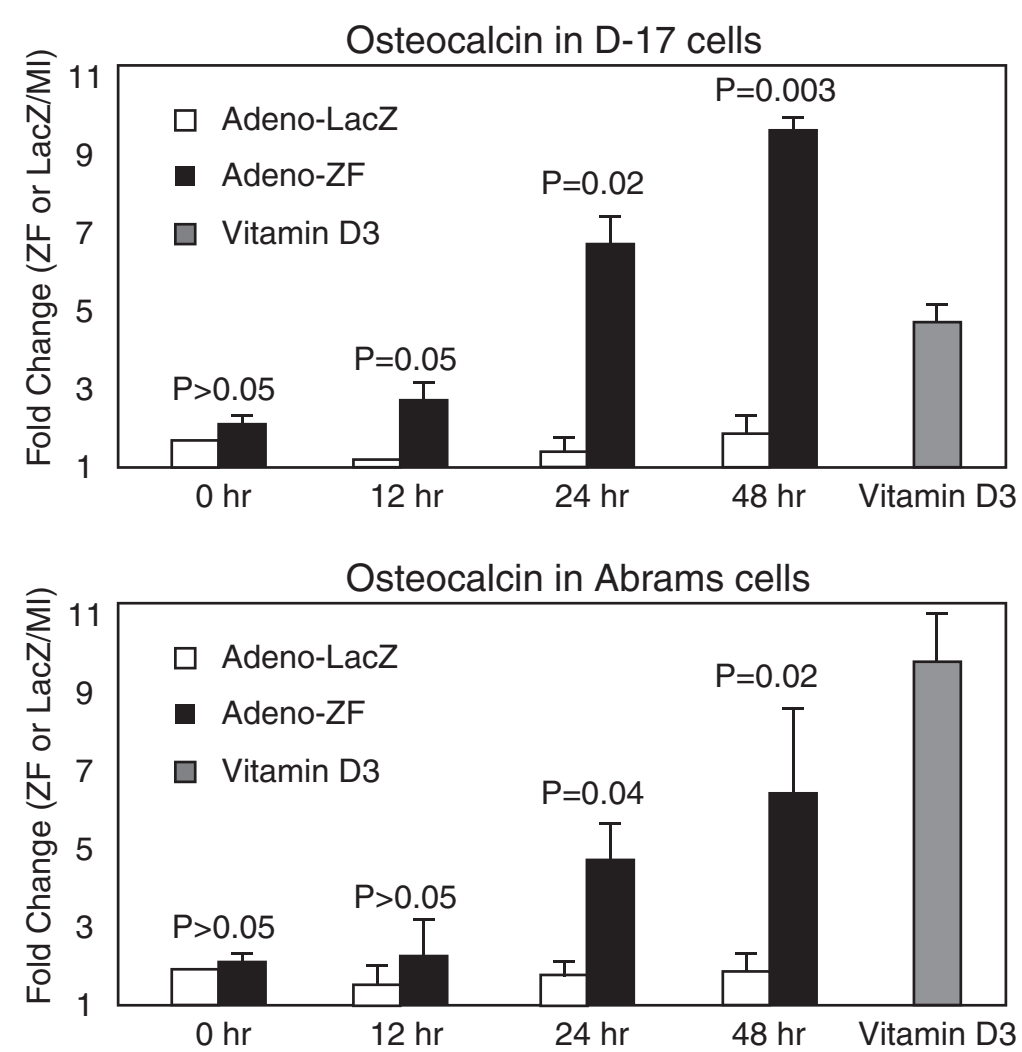

Figure 4 Zhangfei induces differentiation of canine osteosarcoma cells. D-17 and Abrams cells were either mock-infected or infected with Adeno-ZF or Adeno-LacZ. The positive control cells were treated with $10^{-5} \mathrm{mM}$ vitamin D3. The mRNA levels of osteoblast differentiation marker (osteocalcin) were estimated by qRT-PCR. Standard deviations from means and P values as calculated using a Student T-test are shown.

naturally develop therapy resistance and metastasis. In addition, tumor burdens in spontaneously arising cancers of dogs are more similar to humans than the experimentally induced tumors found in murine models, which may be important with regard to biologic factors such as hypoxia and clonal variation. The size of canine tumors also allows for serial imaging and tissue collection over time [29].

In previous studies, we found that the transcription factor CREBZF/Zhangfei suppressed the growth of D-17 dog OS cells [5]. Herein, we further discovered that the growth suppressive effects of Zhangfei were applicable to three other independently isolated canine OS cell lines as well.

The unfolded protein response (UPR) is an adaptive response induced by endoplasmic reticulum (ER) stress, which alleviates ER stress by up-regulating the expression of ER-resident chaperons [30], inducing ER-associated protein degradation (ERAD), and down-regulating the synthesis of new proteins [31,32]. If these mechanisms are not sufficient to alleviate ER stress, then an apoptosis program is initiated to induce cell death. Our previous results [15] suggest that Zhangfei is a potential regulator of the UPR, and it might accelerate UPR feedback mechanisms by interacting with the UPR mediator-Xbp1 and targeting it for proteasomal degradation. In the present study, the strong inhibitory effects of Zhangfei on both pharmacological (thapsigargin) and physiological (glucose deprivation) -induced UPR was also observed in the four canine OS cell lines we examined.

Zhangfei suppresses the UPR and cell growth by stabilizing the tumour suppressor protein p53 [6]. All four canine OS cell lines we examined express functional p53 (Figure 1). These results suggest that the induction of Zhangfei expression in canine OS may be an effective strategy for suppressing cell growth and metastasis. However, the strategy would likely only be successful with OS that have functional p53. A large proportion of human cancers have deleted or otherwise non-functional p53. At present we do not know the proportion of canine OS that have inactive p53. Some studies [33] suggest that most canine OS do not have deletions or major rearrangements in the gene for p53. Although $30-50 \%$ of p53 coding sequences in canine OS have polymorphisms [33-35] the effect of these changes on p53 functionality is unknown. It is therefore difficult to determine how universally applicable Zhangfei would be as a modality for treating canine OS. The role of p53 in canine OS is also 

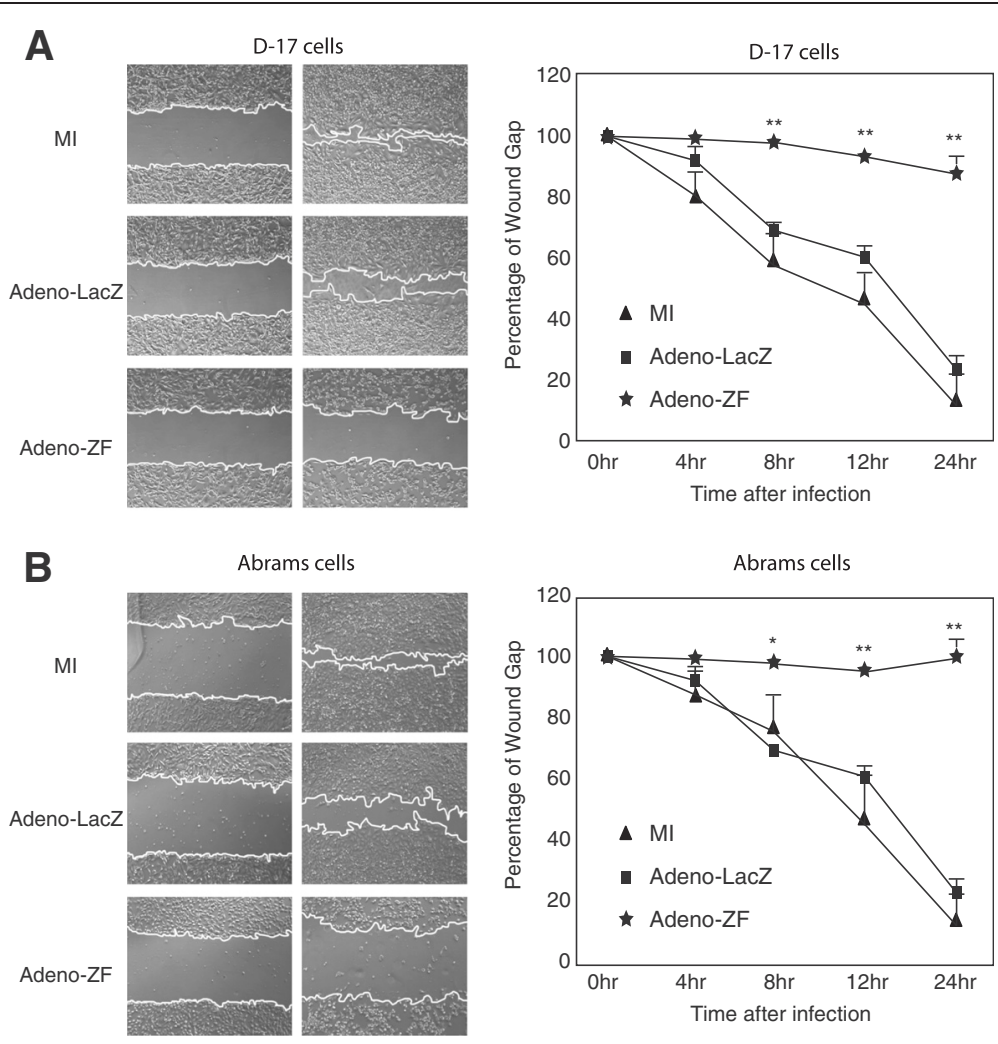

Figure 5 Ectopic expression of Zhangfei causes decreased ability of canine osteosarcoma cells to repair a scratch wound. (A) Scratch wounds were made in 100\% confluent cultures of D-17 or Abrams cells mock-infected or infected with Adeno-ZF or Adeno-LacZ. Phase contrast images were taken at $0,4,8,12$, and 24 hours after infection from identical regions. (B) The wound size relative to the starting wound size was measured at each time point after infection in three independent experiments and expressed as a percentage reduction in wound size + standard deviation. Significance of differences of means $\left({ }^{*} P<0.05,{ }^{* *} P<0.01\right)$ were determined using ANOVA.

controversial. The expression of ectopic p53 in canine OS cells, both in vitro and in vivo models leads to reduced tumour growth and an increase in apoptotic cells [5,36,37]. In contrast, other studies in both humans [38] and dogs $[39,40]$ suggest that increased p53 expression in OS correlates with more aggressive tumours and decreases survival time.

\section{Methods}

\section{Cells and tissue culture}

Canine osteosarcoma D-17 cells, obtained from the American Type Tissue Culture Collection, were grown in MEM-Alpha containing $10 \%$ fetal bovine serum (FBS). Canine Abrams, McKinley and Gracie cell lines were grown in Dulbecco's minimal essential medium containing penicillin, streptomycin and $10 \%$ newborn calf serum. All media, serum and antibiotics were purchased from Invitrogen (Carlsbad, California). Abrams cells were derived from metastatic OS nodules whereas McKinley and Gracie cells were from primary tumours. All cell lines were confirmed to be of canine origin by multispecies multiplex PCR and identified by short tandem repeat analysis as described [41].

\section{Adenovirus Vectors Expressing Zhangfei and B-galactosidase (LacZ)}

These vectors were constructed, grown, and purified using the Adeno-X Expression System (Clontech). They were created in our laboratory as described earlier [14]. Cells were infected with Adeno-Zhangfei, Adeno-LacZ (expressing E. coli ß-galactosidase, LacZ) or mock-infected. A multiplicity of infection (MOI) of 100 plaque-forming units (pfu) per cell was used.

\section{WST-1 cell proliferation and viability assay}

To determine the growth rate of cells, $10^{4}$ cells/well were seeded into 96-well plates. $24 \mathrm{~h}$ later cells were either mock infected or infected with adenovirus vectors expressing Zhangfei (Adeno-ZF) or ß-galactosidase (Adeno-LacZ). Cell proliferation was assessed using Cell Proliferation Reagent WST-1 (Roche, Mannheim, Germany) according to the manufacturer's specifications.

\section{Annexin V-apoptosis assay}

Cells were collected after trypsinization and stained with Annexin $\mathrm{V}$ and propidium iodide (PI) using an Annexin V kit (Calbiochem) following manufacturer's instructions. As 


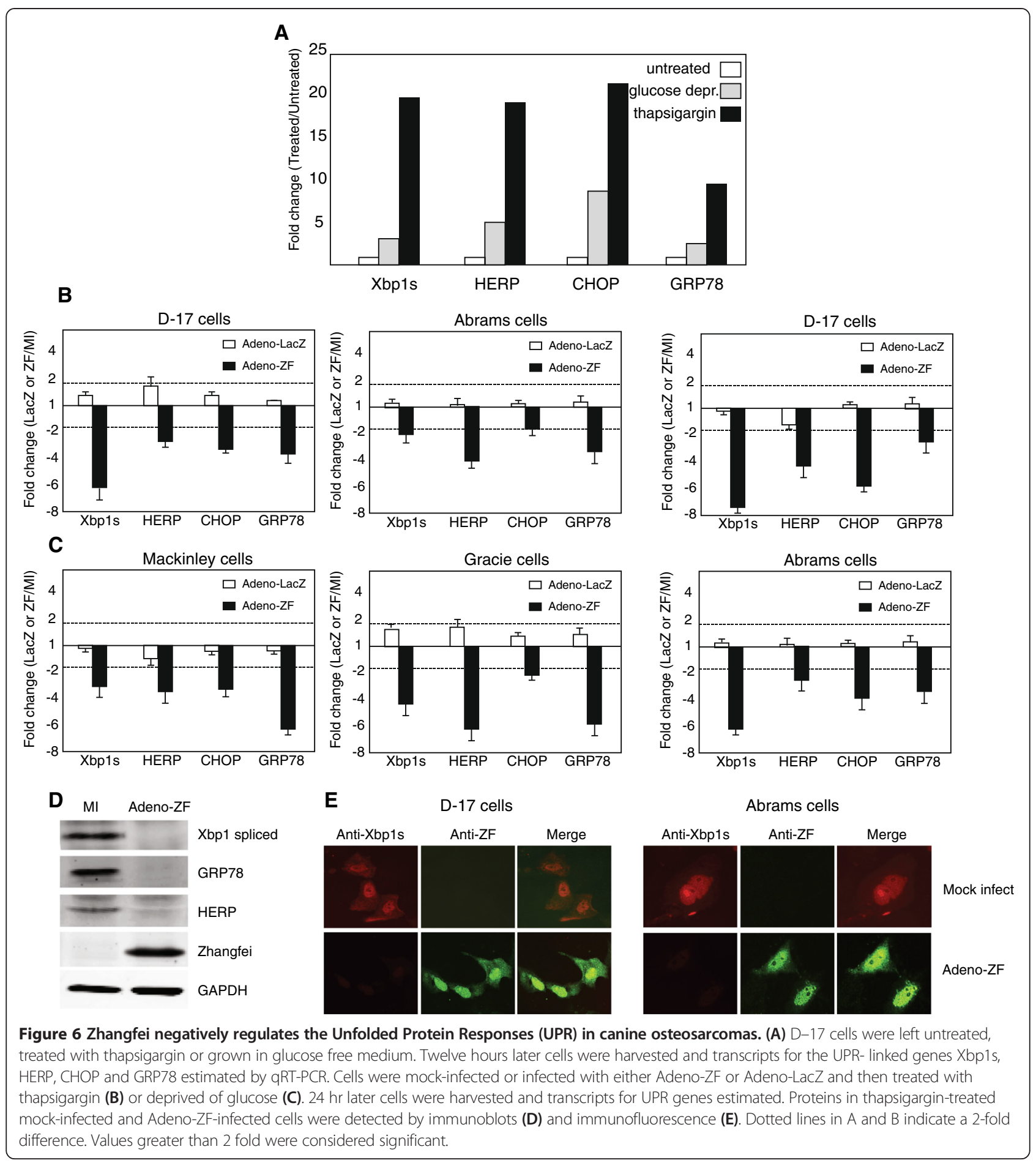

a positive control cells were treated with $50 \mu \mathrm{M}$ etopocide (Calbiochem) for $24 \mathrm{hr}$. Cells were analyzed in a Coulter EPICS XL flow cytometer. In this assay, early apoptotic cells stained with Annexin V but not with PI while late apoptotic or necrotic cells stained with both Annexin V and PI.

\section{Scratch wound healing assay}

Scratch wounds more than $5 \mathrm{~mm}$ in length and of equal thickness were made in $100 \%$ confluent cultures of D-17 or Abrams cells mock-infected or infected with Adeno-ZF or Adeno-LacZ with a $10 \mu \mathrm{L}$ disposable micropipette tip. Phase contrast images were obtained at $0,4,8,12$, and 
24 hours after infection from identical regions. The wound size at each time point after infection relative to the starting wound size was measured using Photoshop software in three independent experiments.

\section{Quantitative real-time PCR (qPCR)}

Total RNA was extracted using RNeasy Plus Mini Kit from Qiagen (Mississauga, ON, Canada). Gene expression was analyzed by RT-PCR using Brilliant II SYBR Green QPCR Master Mix Kit (Agilent Technologies). Levels of GAPDH were used to normalize the samples. All reactions were analyzed in duplicate and each experiment was repeated at least twice. Relative fold changes of transcript levels were calculated as $2^{\Delta \Delta}$ Ct . Nucleotide sequences of the primers used are in Table 1 . The identity of all products was confirmed by electrophoretic mobility on mobility on agarose gels and by determining nucleotide sequence. Only results with homogeneous thermal disassociation profiles were considered.

\section{PCR and sequencing of p53 genes}

The sequences of PCR primers used for canine p53 amplification were: canine p53-forward: GGTGACTG CAATGGAGGAGTCGCA, canine p53-reverse: TCAGT CTGAGTCAAGCCCTTCTCT. RNA was purified from cells using the RNEasy Plus mini kit with a genomic DNA elimination step (Qiagen) and RNA converted to cDNA with the Quantitect Reverse Transcription kit (Qiagen) using instructions supplied by the manufacturer. Two-step RT-PCR reactions used TopTaq emzyme (Qiagen) and were performed in a PCR machine. Sequences were aligned using the software, MacVector. Nucleotide sequences for coding sequences for p53 transcripts recovered from D17, Abrams, McKinley and Gracie cell lines were submitted to GenBank/NCBI and were assigned the following accession numbers: KP279761, KP279762, KP279763, KP279764.

\section{Table 1 Sequence of primers used for qRT-PCR}

\begin{tabular}{lll}
\hline Xbp1 & $\begin{array}{l}\text { spliced-forward } \\
\text { spliced-reverse }\end{array}$ & TCTGCTGAGTCCGCAGCAGG \\
HERP & forward & CCGAGAACTGGGTCCTCT \\
& reverse & CTITGGAAGCAAGTCCTTGA \\
CHOP & forward & TGGAAGCCTGGTATGAGGAC \\
& reverse & TGCCACTTCCTCTCGTCT \\
GRP78 & forward & GGCTTGATAAGAGGGAAGG \\
& reverse & GGTAGAACGGAACAGGTCCA \\
osteocalcin & forward & AAGCRGGAGGGCAGCAGGT \\
& reverse & CYGRTARGCYTCCTGRAAGC \\
GAPDH & forward & TGCCTCCTGCACCACCAACTGC \\
& reverse & GGGCCATCCACAGTCTTCTGGG \\
\hline
\end{tabular}

All sequences are in the $5^{\prime}-3^{\prime}$ direction.
Plasmids and chloramphenicol acetyl transferase (CAT) assay The construction of pcZF [12], a plasmid that expresses Zhangfei in mammalian cells, has been described. The CAT reporter plasmid pCAT3B-p53RE was constructed by transferring oligonucleotides containing two copies of p53 responsive element, GGTCAAGTTGGGACACGTC CaaGAGCTAAGTCCTGACATGTCT (IDT, Coralville, Iowa), to pCAT3Basic (Promega), which contains the coding sequence for CAT linked to a basal promoter. Oligonucleotides representing the p53 responsive elements with overhanging 5 ' terminal KpnI and 3' terminal BglII sites were annealed and ligated to pCAT3Basic cut with the same enzymes.

In CAT assay, D-17, Abrams, Gracie and McKinley cells were transfected with $0.5 \mu \mathrm{g}$ of pCAT3B-p53RE, in the presence or absence of a plasmid expressing Zhangfei (pcZF, $1 \mu \mathrm{g}$ ), using Lipofectamine 2000 (Invitrogen) as described in the manufacturer's instructions. The promoterless parental reporter plasmid, pCAT3B was included as a control to show basal CAT activity. 250 ng of pCMVBGal, a plasmid specifying $\beta$-galactosidase, were added to each transfection as an internal control. 24 h after transfection, the CAT activity was determined by ELISA. CAT values were normalized to $\beta$-galactosidase.

\section{Antibodies, immunoblotting and immunofluorescence}

The antibodies used were mouse anti-FLAG (Sigma), rabbit anti-Zhangfei serum [12], rabbit anti-Xbp1 (Abcam, Cambridge, MA), rabbit anti-HERP (Abcam, Cambridge, MA), rabbit anti-GRP78 (Abcam, Cambridge, MA), and mouse anti-GAPDH (Chemicon, Billerica, MA). Suppliers of antibodies against Xbp1, HERP, GRP78 and GAPDH indicated that they recognized canine proteins. Secondary antibodies were goat anti-mouse Alexa488, goat antirabbit Alexa546 and goat anti-rabbit Cy5 (Invitrogen). Cells were processed for immunoblotting and immunofluorescence as described previously $[5,12]$.

\section{Statistical analysis}

Statistical analysis of data was performed by Student $T$-test or ANOVA using IBM SPSS statistics version 21.0.0 software. ANOVA tests with LSDpost hoc comparison was used to analyze the differences between multi-group means and their associated procedures by adding individuals as a treatment variable, and a paired $T$-test was used to evaluate the effects of one treatment compared with no treatment/control. A P value of less than 0.05 was considered to be statistically significant for both tests.

\section{Availability of supporting data}

Accession numbers sequences supporting the results of this article are available in the GenBank repository, [GenBank: KP279761, GenBank:KP279762, GenBank:KP279763, GenBank:KP279764].” 


\section{Ethics and biosafety statement}

All experiments were done following biosafety procedures and precautions approved by the University of Saskatchewan Biosafety committee and under Biosafety Permit VMB-03. All experiment were done on established cell lines and no animals were used.

\begin{abstract}
Abbreviations
bLZip: Basic leucine zipper; CERBZF: Cyclic AMP response element binding protein Zhangfei; UPR: Unfolded protein response; OS: Osteosarcoma; p53: 53,000 molecular weight protein; Xbp1: X-box binding protein one; ATF4: Activation transcription factor four; SMAD: Homologue of small body size (C. elegans) and mothers against decapentaplegic (Drosophila); VP16: Herpes simplex virus virion protein sixteen; LacZ: Beta galactosidase; GRP78: Glucose responsive protein 78,000 molecular weight; HERP: Homocysteine-responsive endoplasmic reticulum resident protein; CHOP: C/EBP homologous protein; ERAD: Endoplasmic reticulum resident protein degradation; FBS: Foetal bovine serum; MOl: Multiplicity of infection; PI: Propidium iodide; GAPDH: Glyceraldehyde 3-phosphate dehydrogenase; QPCR: Quantitative polymerase chain reaction; CAT: Chloramphenicol acetyl transferase.
\end{abstract}

\section{Competing interests}

The authors declare that they have no competing interests.

\section{Authors' contributions}

RZ, VM and DHT conceived of the experiments and DHT provided the cell lines. RZ performed all the experiments and prepared the initial manuscript and figures. VM, DHT and RZ edited, approved and prepared the final manuscript.

\section{Acknowledgements}

This work was supported by a Discovery grant to VM from the Natural Sciences and Engineering Research Council (NSERC) of Canada and a grant from the Western College of Veterinary Medicine (WCVM) Companion Animal Research Fund and the Kaye Canine Foundation. RZ was supported by scholarships from the Government of China (China Scholarship Council, RZ-2010635007) and the University of Saskatchewan College of Graduate Studies and Research.

\section{Author details}

'Department of Microbiology, Western College of Veterinary Medicine, University of Saskatchewan, Saskatoon, SK, Canada. ${ }^{2}$ Flint Animal Cancer Center, Colorado State University, Fort Collins, CO, USA. ${ }^{3}$ Present address: Department of Basic Veterinary Medicine, College of Veterinary Medicine, China Agricultural University, Beijing, China.

Received: 10 September 2014 Accepted: 20 January 2015

Published online: 07 February 2015

\section{References}

1. Withrow SJ, Wilkins RM. Cross talk from pets to people: translational osteosarcoma treatments. ILAR J. 2010;51(3):208-213.

2. Mueller F, Fuchs B, Kaser-Hotz B. Comparative biology of human and canine osteosarcoma. Anticancer Res. 2007:27(1A):155-164.

3. Khanna C, London C, Vail D, Mazcko C, Hirschfeld S. Guiding the optimal translation of new cancer treatments from canine to human cancer patients. Clin Cancer Res. 2009;15(18):5671-5677.

4. Paoloni M, Khanna C. Translation of new cancer treatments from pet dogs to humans. Nat Rev Cancer. 2008:8(2):147-156.

5. Bergeron T, Zhang R, Elliot K, Rapin N, MacDonald V, Linn K. The effect of Zhangfei on the unfolded protein response and growth of cells derived from canine and human osteosarcomas. Vet Comp Oncol. 2013;11(2):140-50.

6. Zhang R, Misra V. Effects of cyclic AMP response element binding proteinZhangfei (CREBZF) on the unfolded protein response and cell growth are exerted through the tumor suppressor p53. Cell Cycle. 2014;13(2):279-92.

7. Legare ME, Bush J, Ashley AK, Kato T, Hanneman WH. Cellular and phenotypic characterization of canine osteosarcoma cell lines. J Cancer. 2011;2:262-270
8. MacEwen EG, Kutzke J, Carew J, Pastor J, Schmidt JA, Tsan R, et al. c-Met tyrosine kinase receptor expression and function in human and canine osteosarcoma cells. Clin Exp Metastasis. 2003;20(5):421-430.

9. MacEwen EG, Pastor J, Kutzke J, Tsan R, Kurzman ID, Thamm DH, et al. IGF-1 receptor contributes to the malignant phenotype in human and canine osteosarcoma. J Cell Biochem. 2004:92(1):77-91.

10. Schwartz AL, Custis JT, Harmon JF, Powers BE, Chubb LS, LaRue SM, et al. Orthotopic model of canine osteosarcoma in athymic rats for evaluation of stereotactic radiotherapy. Am J Vet Res. 2013;74(3):452-458.

11. Maeda J, Yurkon CR, Fujisawa H, Kaneko M, Genet SC, Roybal EJ, et al. Genomic instability and telomere fusion of canine osteosarcoma cells. PLoS One. 2012;7(8):e43355.

12. Lu R, Misra V: Z. Second cellular protein interacts with herpes simplex virus accessory factor HCF in a manner similar to Luman and VP16. Nucleic Acids Res. 2000;28(12):2446-2454.

13. Cockram GP, Hogan MR, Burnett HF, Lu R. Identification and characterization of the DNA-binding properties of a Zhangfei homologue in Japanese pufferfish, Takifugu rubripes. Biochem Biophys Res Commun. 2006;339(4):1238-1245.

14. Misra V, Rapin N, Akhova $O$, Bainbridge M, Korchinski P. Zhangfei is a potent and specific inhibitor of the host cell factor-binding transcription factor Luman. J Biol Chem. 2005:280(15):15257-15266.

15. Zhang R, Rapin N, Ying Z, Shklanka E, Bodnarchuk TW, Verge VMK, et al. Zhangfei/CREB-ZF - A Potential Regulator of the Unfolded protein Response. PLoS One. 2013;8(10):e77256.

16. Hogan MR, Cockram GP, Lu R. Cooperative interaction of Zhangfei and ATF4 in transactivation of the cyclic AMP response element. FEBS Lett. 2006;580(1):58-62.

17. Lee JH, Lee GT, Kwon SJ, Jeong J, Ha YS, Kim WJ, et al. CREBZF, a novel Smad8-binding protein. Mol Cell Biochem. 2012;368(1-2):147-153.

18. Akhova O, Bainbridge M, Misra $V$. The neuronal host cell factor-binding protein Zhangfei inhibits herpes simplex virus replication. J Virol. 2005:79(23):14708-14718.

19. Lopez-Mateo I, Villaronga MA, Llanos S, Belandia B. The transcription factor CREBZF is a novel positive regulator of p53. Cell Cycle. 2012;11(20):3887-3895.

20. Joerger AC, Fersht AR. Structural biology of the tumor suppressor p53. Annu Rev Biochem. 2008;77:557-582

21. Ciovacco WA, Goldberg CG, Taylor AF, Lemieux JM, Horowitz MC, Donahue $\mathrm{HJ}$, et al. The role of gap junctions in megakaryocyte-mediated osteoblast proliferation and differentiation. Bone. 2009;44(1):80-86.

22. Selvarajah GT, Kirpensteijn J. Prognostic and predictive biomarkers of canine osteosarcoma. Vet J. 2010;185(1):28-35.

23. Fan TM, Charney SC, de Lorimier LP, Garrett LD, Griffon DJ, Gordon-Evans WJ, et al. Double-blind placebo-controlled trial of adjuvant pamidronate with palliative radiotherapy and intravenous doxorubicin for canine appendicular osteosarcoma bone pain. J Vet Intern Med. 2009;23(1):152-60.

24. Tomlin JL, Sturgeon C, Pead MJ, Muir P. Use of the bisphosphonate drug alendronate for palliative management of osteosarcoma in two dogs. Vet Rec. 2000;147(5):129-132

25. Walter CU, Dernell WS, LaRue SM, Lana SE, Lafferty MH, LaDue TA, et al, Curative-intent radiation therapy as a treatment modality for appendicular and axial osteosarcoma: a preliminary retrospective evaluation of 14 dogs with the disease. Vet Comp Oncol. 2005;3(1):1-7.

26. Brodey RS, Abt DA. Results of surgical treatment in 65 dogs with osteosarcoma. J Am Vet Med Assoc. 1976;168(11):1032-1035.

27. Kurzman ID, MacEwen EG, Rosenthal RC, Fox LE, Keller ET, Helfand SC, et al Adjuvant therapy for osteosarcoma in dogs: results of randomized clinical trials using combined liposome-encapsulated muramyl tripeptide and cisplatin. Clin Cancer Res. 1995;1(12):1595-1601.

28. Dow S, Elmslie R, Kurzman I, MacEwen G, Pericle F, Liggitt D. Phase I study of liposome-DNA complexes encoding the interleukin-2 gene in dogs with osteosarcoma lung metastases. Hum Gene Ther. 2005;16(8):937-946.

29. Paoloni M, Davis S, Lana S, Withrow S, Sangiorgi L, Picci P, et al. Canine tumor cross-species genomics uncovers targets linked to osteosarcoma progression. BMC Genomics. 2009;10:625.

30. Kaufman RJ, Scheuner D, Schroder M, Shen X, Lee K, Liu CY, et al. The unfolded protein response in nutrient sensing and differentiation. Nat Rev Mol Cell Biol. 2002;3(6):411-421.

31. Meusser B, Hirsch C, Jarosch E, Sommer T. ERAD: the long road to destruction. Nat Cell Biol. 2005;7(8):766-772.

32. Lai $E$, Teodoro $T$, Volchuk A. Endoplasmic reticulum stress: signaling the unfolded protein response. Physiology. 2007;22:193-201. 
33. Mendoza S, Konishi T, Dernell WS, Withrow SJ, Miller CW. Status of the p53, $\mathrm{Rb}$ and MDM2 genes in canine osteosarcoma. Anticancer Res. 1998;18(6A):4449-4453.

34. van Leeuwen IS, Cornelisse CJ, Misdorp W, Goedegebuure SA, Kirpensteijn J, Rutteman GR. P53 gene mutations in osteosarcomas in the dog. Cancer Lett. 1997;111(1-2):173-178.

35. Johnson AS, Couto CG, Weghorst CM. Mutation of the p53 tumor suppressor gene in spontaneously occurring osteosarcomas of the dog. Carcinogenesis. 1998;19(1):213-217.

36. Kanaya N, Yazawa M, Goto-Koshino Y, Mochizuki M, Nishimura R, Ohno K, et al. Anti-tumor effect of adenoviral vector-mediated p53 gene transfer on the growth of canine osteosarcoma xenografts in nude mice. J Vet Med Sci. 2011:73(7):877-883.

37. Yazawa M, Setoguchi A, Hong SH, Uyama R, Nakagawa T, Kanaya N, et al. Effect of an adenoviral vector that expresses the canine p53 gene on cell growth of canine osteosarcoma and mammary adenocarcinoma cell lines. Am J Vet Res. 2003:64(7):880-888

38. Fu HL, Shao L, Wang Q, Jia T, Li M, Yang DP. A systematic review of p53 as a biomarker of survival in patients with osteosarcoma. Tumour Biol. 2013;34(6):3817-3821.

39. Sagartz JE, Bodley WL, Gamblin RM, Couto CG, Tierney LA, Capen CC. p53 tumor suppressor protein overexpression in osteogenic tumors of dogs. Vet Pathol. 1996;33(2):213-221.

40. Loukopoulos P, Thornton JR, Robinson WF. Clinical and pathologic relevance of p53 index in canine osseous tumors. Vet Pathol. 2003:40(3):237-248

41. O'Donoghue LE, Rivest JP, Duval DL. Polymerase chain reaction-based species verification and microsatellite analysis for canine cell line validation. J Vet Diagn Invest. 2011;23(4):780-785.

\section{Submit your next manuscript to BioMed Central and take full advantage of:}

- Convenient online submission

- Thorough peer review

- No space constraints or color figure charges

- Immediate publication on acceptance

- Inclusion in PubMed, CAS, Scopus and Google Scholar

- Research which is freely available for redistribution 